\title{
Addressing Medication Errors Involving HIV-Positive Inpatients: Development of a Clinician's Guide to Assessing Antiretroviral Therapy
}

\author{
Elliot S Pittman, Emily H Li, and Michelle M Foisy
}

\section{INTRODUCTION}

S ince the advent of combination antiretroviral (ARV) therapy $S$ over 20 years ago, the treatment of HIV-positive patients has been revolutionized. Six classes of ARVs and more than 25 marketed drugs are now available in a variety of combination tablets and formulations, allowing for many treatment options. When treated effectively, HIV infection is now considered a manageable chronic disease, and patients generally have normal life expectancy. ${ }^{1}$ However, with the successes of HIV treatment come new challenges for health care systems and providers. Health care workers in this area often manage multiple co-infections and comorbidities, psychosocial and addiction hardships, and increasingly complex HIV therapies. In addition, in order to achieve lasting virologic suppression and avoid the development of drug resistance and treatment failure, patients must be highly adherent to multiclass combination therapy. ${ }^{2}$ As a result, health care providers must also invest significant time, effort, and creativity as they journey with the patient through the course of lifelong therapy.

Compounding these issues is the challenge of maintaining seamless care when patients transition through various institutional settings such as addiction recovery centres, correctional facilities, and hospitals. Because of the complexity of ARV therapy and the fact that clinicians without specialized training in HIV or infectious diseases are often unfamiliar with HIV management, very high rates of ARV drug errors in HIV-positive inpatients have been reported in the literature. ${ }^{3-27}$ In one study addressing the root causes of error, prescribers and other health care providers admitted that a lack of medication knowledge was a main reason for drug error. ${ }^{10}$ The second most commonly cited reason was lack of accurate medication reconciliation. ${ }^{10}$
Investigators have trialled a number of interventions in efforts to reduce drug errors in the hospital setting. ${ }^{3-18}$ Given that the causes of drug error are usually multifactorial, the solutions must also be multipronged. One of the most successful intervention studies used a combination of staff education, revisions to the computerized order-entry system, and dedication of an HIVtrained pharmacist to conduct medication reconciliation and daily medication reviews. ${ }^{5}$

Although a multifaceted approach to solving this problem is the ideal, it may not be feasible to implement the full complement of interventions in a given institution because of financial and time constraints. Recognizing the significance of medication errors in the hospital setting, the resource limitations faced by health care systems, and the need for staff education, we felt that a comprehensive and accessible guide to ARV therapy designed for inpatient pharmacists and other clinicians would be a useful and educational clinical tool. The objective of this project was to address the common types of ARV-related errors reported in hospitalized HIV-positive patients by creating an evidence-based guide for clinicians that provides both a framework for patient assessment and a collection of useful resources to facilitate clinical decisions. Ultimately, widespread awareness and use of the guide may assist in minimizing drug-related errors in this vulnerable and complex patient population.

\section{DEVELOPMENT OF THE GUIDE}

The patient assessment portion of the guide was based on findings from a recent literature review that summarized drug errors in hospitalized HIV-positive patients and discussed interventions to reduce error. ${ }^{28,29}$ The guide was adapted from a 
framework for patient care processes developed by the University of Alberta Faculty of Pharmacy and Pharmaceutical Sciences ${ }^{30}$ and was customized to address common drug-related errors in HIV inpatients. Supplementary material derived from a variety of key resources provides basic information on available ARV agents, drug interactions, and laboratory investigations and lists handy resources and local contact information. A single-page ARV assessment form, which serves as a companion to the guide, was also created for efficient use in a clinical setting. Several HIV pharmacists, regional pharmacy clinical practice leaders, and an inpatient pharmacist focus group participated in 2 peer-review cycles of the guide for content, readability, and applicability. The complete assessment guide ${ }^{31}$ with supplementary materials was then posted on the institutional website for widespread access (www.bugsanddrugs.ca/documents/HIVARV Guide.pdf).

\section{DESCRIPTION OF THE GUIDE}

The core of the assessment guide consists of a 3-step patient assessment framework, which includes HIV-specific content for each section (Appendix 1, available at www.cjhp-online.cal index.php/cjhp/issue/view/112/showToc). The steps of the assessment process follow the path a patient would take during the hospital stay. Seamless care and communication with the outpatient HIV team are stressed, particularly on admission and discharge. The following sections summarize the steps in the assessment process.

\section{Patient Assessment Process for Antiretrovirals}

Step 1-Assessment on admission: Part A of step 1 consists of creating a patient database, including medical history, social history, and results of pertinent laboratory investigations, and then performing a medication history and reconciliation. Part B of step 1 outlines an assessment framework for ARV therapy, which includes assessment of indication, efficacy, safety, and adherence (see Appendix 1, Step 1).

Step 2-Assessment during hospital stay: Step 2 addresses points in care when ARV medication errors often occur, as reported in the literature. This step includes a daily assessment of medications or whenever medication changes are made, monitoring for common errors during unit transitions (such as omissions, dosing, interactions, scheduling, and auto-stops), and, finally, appropriate monitoring of ARV safety and efficacy (see Appendix 1, Step 2).

Step 3-Assessment on discharge: Step 3 addresses common problems at discharge that could result in suboptimal outpatient ARV adherence and fragmented care. This step includes review of discharge prescriptions, coordination of outpatient ARV dispensing and coverage, adherence with medication therapy, and outpatient follow-up (see Appendix 1, Step 3).

\section{Supplementary Material}

The guide ${ }^{31}$ includes supplementary information, in the form of 7 appendices, to enhance its utility and to direct the user to additional resources. The 7 appendices (available online at www.bugsanddrugs.ca/documents/HIVARVGuide.pdf) cover the following topics:

- HIV laboratory tests: description of each test, along with its indication, normal range for test values, and monitoring frequency

- Drug interactions: summary of significant drug interactions and their mechanisms

- Available ARV agents: name, formulation, dose, renal/ hepatic dose adjustment, food concerns, and side effects for each agent currently available

- Fixed-dose combination ARV products: name, composition, dose, and renal/hepatic dose adjustment for each combination product currently available

- Handy resources: hyperlinks to key HIV websites

- Contact information: phone and fax numbers of HIV clinics, clinicians, and outpatient dispensaries in Alberta

- ARV assessment form: a worksheet that serves as a companion to the guide, to assist clinicians in the assessment process and in their communication with outpatient caregivers (see Appendix 2, available at www.cjhp-online.ca/index. $\mathrm{php/cjhp/issue/view/112/showToc).}$

\section{IMPLICATIONS AND SIGNIFICANCE FOR PRACTICE}

In a recent literature review, we found that the most successful studies addressing medication errors in HIV-positive inpatients included multiple interventions. ${ }^{28,29}$ In these studies, the successful interventions involved medication reconciliation performed within 24 hours of admission, preferably by a trained HIV or infectious diseases pharmacist, coupled with continual monitoring throughout the hospital stay and at discharge. ${ }^{5}$ Other significant strategies involved technology, formulary alerts and updates, and education of the health care team. ${ }^{26}$

Given that specialized HIV and infectious diseases pharmacists may not be available at all hospitals to perform medication reconciliation, other models of care are often employed. For instance, a trained and supported nonspecialist clinical pharmacist may also assist in decreasing medication errors in this population. ${ }^{3,4,10,12,15,18}$ It is paramount, however, that nonspecialist pharmacists receive adequate education about HIV-related disease and ARV medications and have access to appropriate resources and ongoing mentorship.

Pharmacists in the inpatient and outpatient settings within the authors' institution strive to provide seamless care for admitted patients. For example, pharmacists in the inpatient unit notify the HIV outpatient clinic about admitted patients, which allows the ambulatory HIV pharmacist to assist the inpatient unit 
pharmacists in performing medication reconciliation on admission and supporting patient care throughout the hospital stay and at discharge. ${ }^{29}$ Despite this model of care, ARV errors are frequently observed at the time of admission, when ARVs are first prescribed. In some cases, there is also a lack of timely and effective medication reconciliation and a failure to initiate the seamless care process by involving the outpatient HIV team in a timely manner. There have been issues with unidentified drug interactions during the hospital stay and drug errors at the time discharge prescriptions are written. These anecdotal observations reflect problems that have been reported in the literature. ${ }^{28}$

Although efforts to reduce drug error are multifaceted, this project has addressed the educational needs of nonspecialist clinicians who may have limited experience dealing with HIV disease and treatment. The ARV assessment guide focuses on problem areas that arise as patients transition throughout the hospital stay and provides a thought process and framework to alert clinicians to these problems. To the authors' knowledge, no other published clinical tools exist to assist clinicians in preventing, identifying, and managing ARV-related problems in the hospital setting. This ARV assessment guide is therefore unique and can serve as a valuable interdisciplinary educational tool for students, residents, and nonspecialist clinicians. The guide has wide applicability and is readily adaptable to other institutions or practice settings. The process and framework used to develop the guide are also transferable to other specialized disease states for which pharmacists may have limited drug knowledge.

A number of educational initiatives have been undertaken to promote the guide within the authors' institution. Pharmacist focus-group discussions have served to educate inpatient pharmacists and have highlighted both opportunities for and barriers to use of the guide in practice. These sessions have also served as a catalyst for discussion of the implementation of other initiatives to reduce drug error, such as technologic support and improved communication. The guide has also been presented to physician prescribers who commonly work with HIV-positive inpatients (e.g., physicians and residents specializing in internal medicine, infectious diseases, and critical care).

The guide will be updated annually to reflect current practice and available ARV agents. Future initiatives relating to the guide may include studying its impact on the number of drug-related errors in the HIV-positive inpatient population. Making the guide available through alternative delivery platforms, such as a pocket card or smartphone application, may enhance its overall accessibility. The guide currently focuses solely on ARV therapy, but expansion of its content to cover prophylaxis and treatment of opportunistic infections seen in this patient population may also be useful.

\section{CONCLUSION}

Given the high rates of medication errors reported among HIV-positive inpatients, an evidence-based clinical guide for the assessment of HIV pharmacotherapy for inpatients was developed for nonspecialist clinicians. According to the literature, the most significant reductions in medication errors in this population have involved a multifaceted approach, including education of health care workers. This clinical guide incorporates such an approach.

The assessment guide was designed to address the educational needs of clinicians who may require additional knowledge and support when caring for HIV-positive inpatients. With the growing number of ARVs and the increasing complexity of therapy, we hope that the guide will increase awareness of the types of medication errors typically observed in this population and enable pharmacists and other clinicians to optimize drugrelated outcomes for their patients. The guide has wide applicability and is easily adaptable to other institutions or practice settings. Although this HIV assessment guide serves as a starting point for clinicians, ongoing vigilance and education will be needed as HIV treatment advances lead to increasing regimen complexity and new ARVs and drug formulations.

\section{References}

1. Tseng A, Foisy M, Hughes CA, Kelly D, Chan S, Dayneka N, et al. Role of the pharmacist in caring for patients with HIV/AIDS: clinical practice guidelines. Can J Hosp Pharm. 2012;65(2):125-45.

2. Panel on Antiretroviral Guidelines for Adults and Adolescents. Guidelines for the use of antiretroviral agents in HIV-1-infected adults and adolescents. Rockville (MD): Department of Health and Human Services; 2015 Apr 8 [cited 2015 Jun 10]. Available from: www.aidsinfo.nih.gov/guidelines/html/ 1/adult-and-adolescent-arv-guidelines/0/

3. Eginger KH, Yarborough LL, Inge LD, Basile SA, Floresca D, Aaronson PM. Medication errors in HIV-infected hospitalized patients: a pharmacist's impact. Ann Pharmacother. 2013;47(7-8):953-60.

4. Lauzevis S, Chaix F, Lazzerini C. Evaluation of a strategy aimed at reducing errors in antiretroviral prescriptions for hospitalized HIV-infected patients. Med Mal Infect. 2013;43(9):391-7.

5. Daniels LM, Raasch RH, Corbett AH. Implementation of targeted interventions to decrease antiretroviral-related errors in hospitalized patients. Am J Health Syst Pharm. 2012;69(5):422-30.

6. Holtzman CW, Gallagher JC. Antiretroviral medication errors among hospitalized HIV-infected adults. Clin Infect Dis. 2012;55(11):1585-6.

7. Liao TV, Rab S, Armstrong WS. Evaluation of medication errors in patients infected with human immunodeficiency virus treated with antiretroviral therapy. Am J Health Syst Pharm. 2012;69(17):1461-2.

8. Yehia BR, Mehta JM, Ciuffetelli D, Moore RD, Pham PA, Metlay JP, et al. Antiretroviral medication errors remain high but are quickly corrected among hospitalized HIV-infected adults. Clin Infect Dis. 2012;55(4):593-9.

9. Carcelero E, Tuset M, Martin M, De Lazzari E, Codina C, Miró J, et al. Evaluation of antiretroviral-related errors and interventions by the clinical pharmacist in hospitalized HIV-infected patients. HIV Med. 2011;12(8): 494-9.

10. Snyder AM, Klinker K, Orrick JJ, Janelle J, Winterstein AG. An indepth analysis of medication errors in hospitalized patients with HIV. Ann Pharmacother. 2011;45(4):459-68.

11. Corrigan MA, Atkinson KM, Sha BE, Crank CW. Evaluation of pharmacyimplemented medication reconciliation directed at antiretroviral therapy in hospitalized HIV/AIDS patients. Ann Pharmacother. 2010;44(1):222-3.

12. Horace AE, Philips M. Identification and prevention of antiretroviral medication errors at an academic medical center. Hosp Pharm. 2010; 45(12):927-33.

13. Pastakia SD, Corbett AH, Raasch RH, Napravnik S, Correll TA. Frequency of HIV-related medication errors and associated risk factors in hospitalized patients. Ann Pharmacother. 2008;42(4):491-7.

14. Heelon M, Skiest D, Tereso G, Meade L, Weeks J, Pekow P, et al. Effect of a clinical pharmacist's interventions on duration of antiretroviral-related errors in hospitalized patients. Am J Health Syst Pharm. 2007;64(19):2064-8. 
15. Sterling ES, Romanelli F, Martin CA, Hoven AD, Smith KM. Impact of a pharmacy-initiated HIV admission note on medication errors within an academic hospital. Hosp Pharm. 2005;40(10):874-81.

16. Segarra-Newnham M. Preventing medication errors with a "pharmacy admission note" for HIV-positive patients. Hosp Pharm. 2002;37(1):34-7.

17. Garey KW, Teichner P. Pharmacist intervention program for hospitalized patients with HIV infection. Am J Health Syst Pharm. 2000;57(24):2283-4.

18. Purdy BD, Raymond AM, Lesar TS. Antiretroviral prescribing errors in hospitalized patients. Ann Pharmacother. 2000;34(7-8):833-8.

19. Brennan K, Moreau S, Curci M, Grigoriu A, Rao N. Evaluation of antiretroviral prescribing error rates and potentially associated risk factors at hospital discharge: preliminary 12-month results. Pharmacotherapy. 2013;33(5):E77.

20. Commers T, Swindells S, Sayles H, Gross AE, Devetten M, Sandkovsky U. Antiretroviral medication prescribing errors are common with hospitalization of HIV-infected patients. J Antimicrob Chemother. 2014;69(1):262-7.

21. Rao N, Patel V, Grigoriu A, Kaushik P, Brizuela M. Antiretroviral therapy prescribing in hospitalized HIV clinic patients. HIV Med. 2012;13(6): 367-71.

22. Mok S, Minson Q. Drug-related problems in hospitalized patients with HIV infection. Am J Health Syst Pharm. 2008;65(1):55-9.

23. Tourret J, Tostivint I, Tezenas Du Montcel S, Karie S, Launay-Vacher V, Vigneau C, et al. Antiretroviral drug dosing errors in HIV-infected patients undergoing hemodialysis. Clin Infect Dis. 2007;45(6):779-84.

24. Rastegar DA, Knight AM, Monolakis JS. Antiretroviral medication errors among hospitalized patients with HIV infection. Clin Infect Dis. 2006; 43(7):933-8.

25. Gray J, Hicks RW, Hutchings C. Antiretroviral medication errors in a national medication error database. AIDS Patient Care STDS. 2005;19(12): 803-12.

26. Chung P, Guo Y, Abam C. Use of information technology to reduce antiretroviral medical errors in hospitalized patients. Pharmacotherapy. 2013;33(5):E56

27. Holtzman CW, Gallagher JC, Bettiker RL, Samuel R. Impact of outpatient electronic medical record on antiretroviral errors among hospitalized patients. Am J Health Syst Pharm. 2013;70(15):1271-2.

28. Li EH, Foisy MM. Antiretroviral and medication errors in hospitalized HIV-positive patients: a review of the literature. Ann Pharmacother. 2014;48(8):998-1010.

29. Foisy MM, Li EH. Response to comment on "Medication reconciliation by a pharmacist in patients with human immunodeficiency virus on antiretroviral therapy: the needed balance of availability and expertise." Ann Pharmacother. 2014;48(11):1535-6.
30. Patient care process. Version 1.3. Edmonton (AB): University of Alberta, Faculty of Pharmacy and Pharmaceutical Sciences, and Alberta Health Services, Edmonton Zone; 2013 Aug [cited 2015 Jun 10]. Available from: http://pharm.ualberta.ca/-/media/pharm/preceptors/documents/trainingand-resources/pcp-whole-document-updated-august-2013.pdf

31. Pittman E, Foisy M. A guide to assessing antiretroviral therapy in HIV hospitalized patients. Edmonton (AB): Royal Alexandra Hospital, Northern Alberta Program; 2014 Oct 17 [cited 2015 Jun 10]. Available from: www.bugsanddrugs.ca/documents/HIVARVGuide.pdf

Elliot S Pittman, BScPharm, PharmD, was, at the time this project was undertaken, a student in the PharmD program of the Faculty of Pharmacy and Pharmaceutical Sciences, University of Alberta, Edmonton, Alberta. $\mathrm{He}$ is now studying medicine at the Cumming School of Medicine, University of Calgary, Calgary, Alberta.

Emily H Li, BScPharm, PharmD, was, at the time this project was undertaken, a student in the PharmD program of the Faculty of Pharmacy and Pharmaceutical Sciences, University of Alberta, Edmonton, Alberta. She is now a Clinical Pharmacist at the Royal Alexandra Hospital, Edmonton, Alberta.

Michelle M Foisy, BSCPharm, PharmD, ACPR, FSCHP, AAHIVP, is an HIV Clinical Pharmacist with the Northern Alberta Program, Royal Alexandra Hospital, Edmonton, Alberta.

Competing interests: Michelle Foisy has received honoraria for providing continuing education and/or serving as a consultant on advisory boards, and/or has received unrestricted educational grants for academic projects from Bristol-Myers Squibb Canada, Gilead Sciences Canada Inc, Janssen Pharmaceuticals, Merck Canada, and ViiV Healthcare. No other competing interests were declared.

\section{Address correspondence to :}

Dr Michelle M Foisy

Royal Alexandra Hospital

CSC 246, 10240 Kingsway Avenue

Edmonton $\mathrm{AB}$ T5H 3V9

e-mail: Michelle.Foisy@albertahealthservices.ca

Funding: None received. 